\title{
Cross Validation of Spaceborne Radar and Ground Polarimetric Radar Aided by Polarimetric Echo Classification of Hydrometeor Types
}

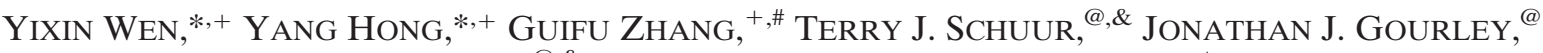 \\ ZAC FlAMig, ${ }^{\circledR \& \&}$ K. RoBERT MORRIS,** AND QING CAO ${ }^{+}$ \\ * Department of Civil Engineering and Environmental Science, University of Oklahoma, Norman, Oklahoma \\ ${ }^{+}$Atmospheric Radar Research Center, University of Oklahoma, Norman, Oklahoma \\ \# School of Meteorology, University of Oklahoma, Norman, Oklahoma \\ ${ }^{\circledR}$ NOAA/National Severe Storms Laboratory, Norman, Oklahoma \\ \& Cooperative Institute for Mesoscale Meteorological Studies, University of Oklahoma, Norman, Oklahoma \\ ** Science Applications International Corporation, and NASA Goddard Space Flight Center, Greenbelt, Maryland
}

(Manuscript received 11 August 2010, in final form 5 January 2011)

\begin{abstract}
Ground-based polarimetric weather radar is arguably the most powerful validation tool that provides physical insight into the development and interpretation of spaceborne weather radar algorithms and observations. This study aims to compare and resolve discrepancies in hydrometeor retrievals and reflectivity observations between the NOAA/National Severe Storm Laboratory "proof of concept" KOUN polarimetric Weather Surveillance Radar-1988 Doppler (WSR-88D) and the spaceborne precipitation radar (PR) on board NASA's Tropical Rainfall Measuring Mission (TRMM) platform. An intercomparison of PR and KOUN melting-layer heights retrieved from 2 to $5 \mathrm{~km}$ MSL shows a high correlation coefficient of 0.88 with relative bias of $5.9 \%$. A resolution volume-matching technique is used to compare simultaneous TRMM PR and KOUN reflectivity observations. The comparisons reveal an overall bias of $<0.2 \%$ between PR and KOUN. The bias is hypothesized to be from non-Rayleigh scattering effects and/or errors in attenuation correction procedures applied to Ku-band PR measurements. By comparing reflectivity with respect to different hydrometeor types (as determined by KOUN's hydrometeor classification algorithm), it is found that the bias is from echoes that are classified as rain-hail mixture, wet snow, graupel, and heavy rain. These results agree with expectations from backscattering calculations at $\mathrm{Ku}$ and $\mathrm{S}$ bands, but with the notable exception of dry snow. Comparison of vertical reflectivity profiles shows that PR suffers significant attenuation at lower altitudes, especially in convective rain and in the melting layer. The attenuation correction performs very well for both stratiform and convective rain, however. In light of the imminent upgrade of the U.S. national weather radar network to include polarimetric capabilities, the findings in this study will potentially serve as the basis for nationwide validation of space-based precipitation products and also invite synergistic development of coordinated space-ground multisensor precipitation products.
\end{abstract}

\section{Introduction}

Reliable quantitative precipitation estimation (QPE) can provide essential information in understanding the water cycle and terrestrial hydrologic processes on a global scale. QPE measured from space with adequate ground validation and calibration can offer consistent and accurate global rainfall information (Chandrasekar et al. 2008). The spaceborne precipitation radar (PR) on

Corresponding author address: Dr. Yang Hong, Dept. of Civil Engineering and Environmental Sciences, University of Oklahoma, Norman, OK 73019.

E-mail: yanghong@ou.edu board the National Aeronautics and Space Administration (NASA) Tropical Rainfall Measuring Mission (TRMM) satellite, launched in late 1997, is the first weather radar to estimate rainfall over the tropics and subtropics from space (Simpson et al. 1996). The PR operating at $\mathrm{Ku}$ band $(13.8 \mathrm{GHz})$ often suffers attenuation that is corrected by a combination of the surface reference and Hitschfeld-Bordan methods (Iguchi et al. 2000). Even though the internal and external calibration of PR confirms that the PR functions properly and has good performance in quantitatively measuring the three-dimensional structure of precipitation (Kozu et al. 2001), cross validation with ground radars (GR) - in particular, those with polarimetric capability—is of vital 
importance to understanding PR measurements and derived products.

A variety of methods have been developed to align spaceborne and ground radar data so as to compare their observations. Schumacher and Houze (2000) compared the area echo coverage detected by PR and GR and found that PR captures the main rain regions but misses weaker echoes. Amitai et al. (2009) performed a comparison between the PR and GR probability distribution functions (pdfs) of the instantaneous rain rate and showed that the pdfs of PR are generally shifted toward lower rain rates. By using resolution matching and the potential geometric distortion correction method, Bolen and Chandrasekar (2003) aligned the two systems and found no systematic difference between the two instruments. Comparisons performed at longer time scales at four TRMM ground validation sites by Wang and Wolff (2009) showed that attenuation is corrected well for convective rain but is slightly overcorrected for stratiform rain. On the other hand, using observations in Melbourne, Florida, Liao and Meneghini (2009) found that $\mathrm{PR}$ attenuation is underestimated in convective rain but is accurately corrected in stratiform rain. In discussing differences between TRMM PR and GR rainfall estimates in prior studies, several reasons are suggested such as calibration differences, poor quality of the GR reference, scattering differences, volume-matching mismatches, uncertain attenuation correction methods, and inaccurate reflectivity-to-rainfall relationships. The current study incorporates hydrometeor classification information from ground-based polarimetric radar to classify the PR-GR comparisons as a function of hydrometeor type.

In conventional rainfall estimation using singlepolarimetric radar accuracy is often limited by frozen or partially frozen hydrometeors (e.g., hail, wet snow, graupel) as well as nonmeteorological scatterers (e.g., ground clutter, birds, or insects). Polarimetric weather radar can significantly improve QPE by identifying rain echoes from other hydrometeor types and as such is a very powerful tool for PR validation (Chandrasekar et al. 2008). Park et al. (2009) developed a polarimetric radar hydrometeor classification algorithm (HCA) that discerns 10 different classes of radar echo: 1) ground clutter or anomalous propagation, 2) biological scatterers, 3) dry aggregated snow, 4) wet snow, 5) crystals of various orientations, 6) graupel, 7) big drops, 8) light and moderate rain, 9) heavy rain, and 10) a mixture of rain and hail. The polarimetric HCA, which includes an automated detection of the bright band, also plays a fundamental role in the polarimetric QPE through a rainfall estimation scheme that varies according to hydrometeor type (Giangrande and Ryzhkov 2008). Furthermore, cross validation of PR with GR with data classified by different hydrometeor type advances the understanding of the root causes of discrepancies. Thus, the dualpolarization HCA plays an important role in this study.

The objective of this study is to compare and resolve discrepancies in hydrometeor and reflectivity observations between the National Severe Storms Laboratory (NSSL) polarimetric prototype of the KOUN Weather Surveillance Radar-1988 Doppler (WSR-88D) and the spaceborne TRMM PR. In section 2 we discuss the data, methods for aligning the sampling volumes, and statistics for comparing PR and GR. Section 3 first presents results for retrieved melting-layer heights observed by TRMM PR and KOUN. Then, results are shown for TRMM PR and KOUN reflectivity comparisons with all data combined. To help to resolve the discrepancies between PR and GR further, reflectivity comparisons are grouped by hydrometeor type and biases are quantified. Vertical profiles with and without attenuation correction for convective and stratiform rain types are compared. A summary and conclusions follow in section 4 .

\section{Data and GR-PR matching methods}

\section{a. NOAA/NSSL KOUN GR and NASA TRMM PR}

In this paper, we use the National Oceanic and Atmospheric Administration (NOAA) NSSL prototype polarimetric radar for the ongoing upgrade of the Next Generation Weather Radar (NEXRAD) national network and NASA's TRMM PR. As a research radar, KOUN has been collecting data on an event-by-event, noncontinuous basis since 2002 . The resolution volume of the KOUN product is $250 \mathrm{~m}$, and angular resolution is about $1^{\circ}$. The volume coverage pattern (VCP) of each event is VCP11, which means the radar completes one volume scan (14 elevation scans) every 5-6 min. We identify 20 TRMM PR overpasses that correspond to coincident overpasses by TRMM PR (Fig. 1a) and meet the following criteria: 1) the maximum time discrepancy between TRMM PR and KOUN observations is less than 3 min, 2) TRMM PR and KOUN meteorologicalecho overlapping areas are larger than $5000 \mathrm{~km}^{2}$ within $150 \mathrm{~km}$ of KOUN, and 3) KOUN data are collected in VCP11. These 20 overpasses, referred to hereinafter as events, are from 14 different days from 2005 to 2009. Note that $\mathrm{P} 1$ and $\mathrm{P} 2$ refer to the first and second coincident overpass on the same day. The event descriptions and times are listed in Table 1. The sixth column lists a subjective quality-control flag for each event. Comments supplied here mention details such as that the TRMM overpass has no bright band detected during event 10 because of intense convection that lacks stratiform precipitation but that the reflectivity product has good quality. 

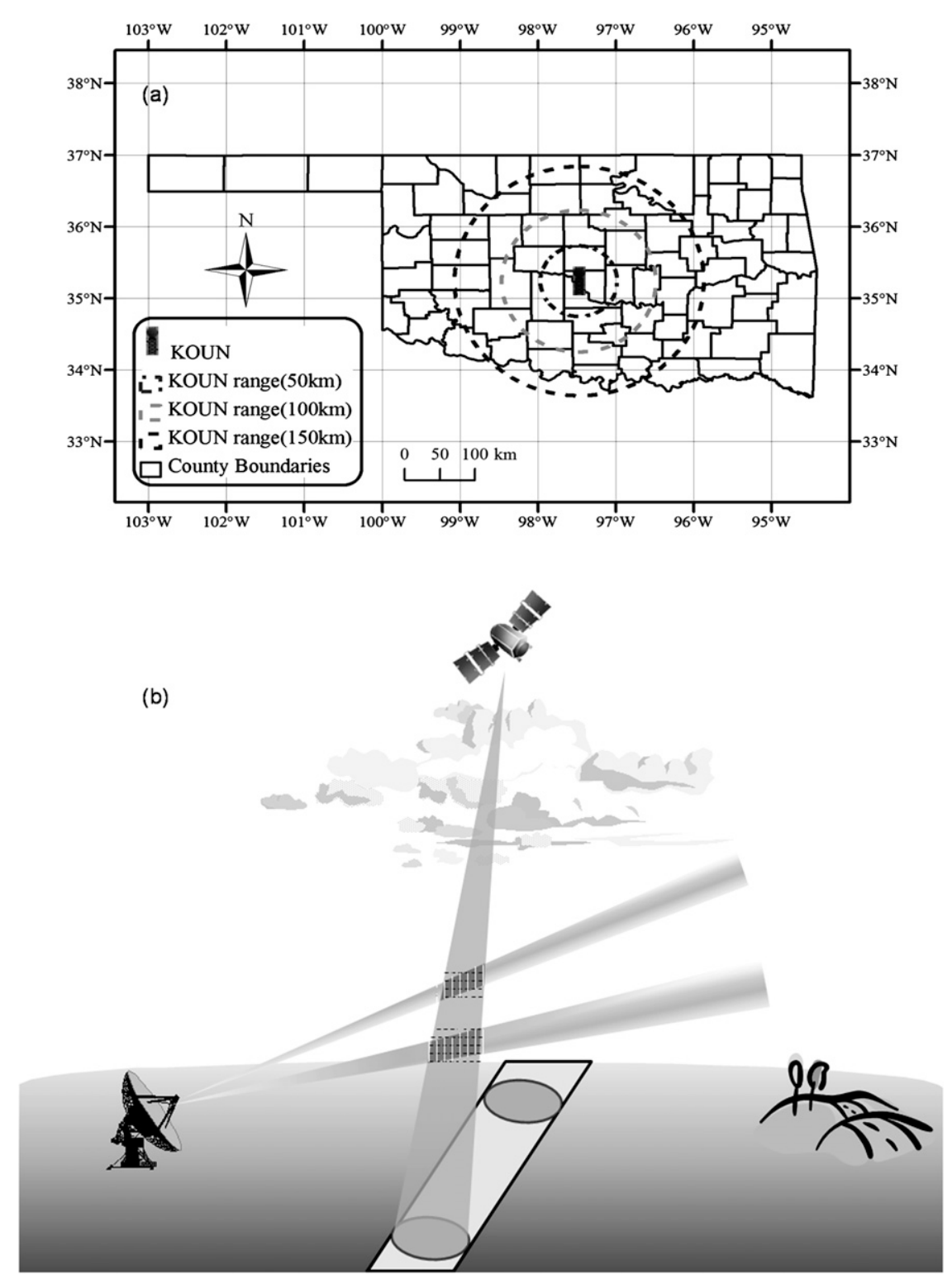

FIG. 1. (a) Study region showing KOUN location and 50-, 100-, and 150-km range rings. (b) Schematic of resolution volume-matching technique of ground-based KOUN and spaceborne TRMM PR. The cross-hatched area on the cones shows the intersections between KOUN and PR sweeps.

There are two cases (events 11 and 15) that have meteorological echoes located at ranges that are greater than $120 \mathrm{~km}$ from KOUN, which will yield greater uncertainty in the KOUN measurements because of nonuniform beam-filling effects. These two events are not considered in reflectivity comparisons in the following analyses but are used in the melting-layer comparisons.

\section{b. Spaceborne PR and GR matching methods}

Diverse approaches have been developed to match PR and GR observations. These approaches can be divided into three categories: 1) comparing the maps of echoes and rain rates to determine the fractional area not detectable by the PR (because of its lower sensitivity) (Schumacher and Houze 2000), 2) resampling PR and GR data to a common 3D Cartesian grid centered on the GR site (Anagnostou et al. 2001; Bolen and Chandrasekar 2000; Liao and Meneghini 2009; Wang and Wolff 2009), and 3) matching PR and GR to the same resolution volume by determining the intersection of the individual PR and GR rays (Bolen and Chandrasekar 2003; Morris and Schwaller 2009). The 
TABLE 1. Description of events used in PR-KOUN comparisons. Times are in UTC. An X quality-control flag indicates good quality of the coincident overpass.

\begin{tabular}{|c|c|c|c|c|c|}
\hline $\begin{array}{c}\text { Event } \\
\text { no. }\end{array}$ & $\begin{array}{l}\text { Events/case } \\
\text { study }\end{array}$ & Description & TRMM time & KOUN time & Quality-control flag \\
\hline 1 & 13 May 2005 & MCS & 0723:37-0726:35 & 0723:10-0728:55 & $\mathrm{X}$ \\
\hline 2 & 6 Oct 2005 (P1) & Widespread rain & 0455:03-0458:01 & 0453:11-0459:36 & $\mathrm{X}$ \\
\hline 3 & 6 Oct $2005(\mathrm{P} 2)$ & Widespread rain & 0632:38-0635:36 & 0631:06-0637:15 & $\mathrm{X}$ \\
\hline 4 & 30 Nov 2006 & Convection, freezing rain, snow & 0744:08-0747:06 & 0741:22-0748:50 & $\mathrm{X}$ \\
\hline 5 & 19 Dec 2006 (P1) & Winter storm & 2123:33-2126:31 & $2121: 48-2127: 50$ & $\mathrm{X}$ \\
\hline 6 & 19 Dec 2006 (P2) & Winter storm & 2301:10-2304:08 & 2259:10-2304:56 & $\mathrm{X}$ \\
\hline 7 & 9 May 2007 & MCS & 0030:51-0033:49 & 0030:14-0034:47 & $\mathrm{X}$ \\
\hline 8 & 24 May 2007 (P1) & Intense convective line, stratiform & $1434: 33-1437: 31$ & 1433:55-1438:29 & $\mathrm{X}$ \\
\hline 9 & 24 May 2007 (P2) & Intense convective line, stratiform & 1612:09-1615:07 & $1611: 25-1615: 58$ & $\mathrm{X}$ \\
\hline 10 & 14 Jun 2007 (P1) & MCS, intense convection & 0452:04-0455:02 & 0451:16-0455:49 & No bright band detected \\
\hline 11 & 14 Jun 2007 (P2) & MCS, intense convection & 0629:41-0632:39 & 0628:45-0633:17 & $\begin{array}{l}\text { Meteorological echo at } \\
\text { far range from KOUN }\end{array}$ \\
\hline 12 & 20 Jun 2007 & MCS & 0235:16-0238:13 & 0234:35-0239:08 & $\mathrm{X}$ \\
\hline 13 & 26 Jun 2007 & MCS & 0018:13-0021:11 & 0017:10-0021:43 & $\mathrm{X}$ \\
\hline 14 & 28 Jun 2007 (P1) & Scattered showers & 2132:00-2134:58 & 2130:56-2135:29 & $\mathrm{X}$ \\
\hline 15 & 28 Jun 2007 (P2) & Scattered showers & 2309:37-2312:35 & 2310:46-2315:18 & $\begin{array}{l}\text { Meteorological echo at } \\
\text { far range from KOUN }\end{array}$ \\
\hline 16 & $13 \mathrm{Jul} 2007$ & Intense convective line, stratiform & 1410:36-1413:34 & 1410:02-1414:34 & $\mathrm{X}$ \\
\hline 17 & 19 Aug 2007 & Tropical Storm Erin & 1913:08-1916:06 & 1912:12-1916:45 & $\mathrm{X}$ \\
\hline 18 & 9 Jun 2008 (P1) & Intense convective line, stratiform & 1314:56-13:18:16 & $1312: 21-1317: 09$ & $\mathrm{X}$ \\
\hline 19 & 9 Jun 2008 (P2) & Intense convective line, stratiform & 1452:40-1456:00 & 1451:59-1556:45 & $\mathrm{X}$ \\
\hline 20 & 11 Feb 2009 & Supercells & 0902:57-0906:11 & 0901:56-0907:02 & $\mathrm{X}$ \\
\hline
\end{tabular}

resolution volume-matching technique is the algorithm adopted for global precipitation measurement (GPM) Ground Validation System Validation Network software available on a NASA Internet site (http://opensource. gsfc.nasa.gov/projects/GPM/index.php). Our study uses this software package for matching PR and KOUN reflectivity observations. We select each PR ray and KOUN ray within a $150-\mathrm{km}$ range from the KOUN site. Comparisons are performed at all matched bins from 1.5 to $13.5 \mathrm{~km}$. By assuming standard atmospheric refraction, we calculate the height above ground where the PR ray intersects the KOUN rays. Also, we calculate the vertical beamwidth of KOUN and horizontal beamwidth of PR at this range. Matching PR and KOUN sample volumes are computed for each PR ray intersection with a GR sweep surface. The PR matching volumes consist of PR reflectivity gates at $250-\mathrm{m}$ vertical resolution, linearly averaged in the vertical direction between the half-power points of the intersecting GR beams for each GR sweep (see Fig. 1b). The GR matching volumes consist of horizontal averages of GR reflectivity gates surrounding the center of each PR ray intersecting a GR sweep, within the area defined by the half-power points of the PR ray (approximately 5-km diameter). The GR gates are weighted by distance from the PR ray center in the GR averaging scheme, using a Barnes (1973)-type Gaussian weighting. Reflectivity data are averaged in linear units and then are converted to decibels. See Morris and Schwaller (2009) for additional details on the resolution volume-matching technique between PR and GR.

\section{c. Cross-validation indices}

We select four statistical indices for evaluating TRMM PR observations using KOUN as the reference. The Pearson correlation coefficient (CC) is used to assess the agreement between PR and KOUN observations. Relative bias (i.e., the bias in percent) is used to assess the systematic bias of PR observations. The mean absolute error (MAE) measures the average magnitude of the error. The rootmean-square error (RMSE) also measures the average error magnitude but gives greater weight to the larger errors. MAE and RMSE are in units of kilometers for melting-layer height comparisons and in decibels for reflectivity comparisons. The formulas for these indices are

$\mathrm{CC}=\frac{\sum_{i=1}^{N}[\operatorname{KOUN}(i)-\overline{\mathrm{KOUN}}] \sum_{i=1}^{N}[\mathrm{PR}(i)-\overline{\mathrm{PR}}]}{\left\{\sum_{i=1}^{N}[\mathrm{KOUN}(i)-\overline{\mathrm{KOUN}}]^{2} \sum_{i=1}^{N}[\mathrm{PR}(i)-\overline{\mathrm{PR}}]^{2}\right\}^{1 / 2}}$,

bias $=\frac{\sum_{i=1}^{N}\left[\operatorname{PR}(i)-\sum_{i=1}^{N} \operatorname{KOUN}(i)\right]}{\sum_{i=1}^{N} \operatorname{KOUN}(i)} \times 100$, 


$$
\begin{aligned}
\mathrm{MAE} & =\frac{\sum_{i=1}^{N}|\operatorname{PR}(i)-\operatorname{KOUN}(i)|}{N}, \text { and } \\
\mathrm{RMSE} & =\left[\frac{\sum_{i=1}^{N}|\operatorname{PR}(i)-\operatorname{KOUN}(i)|^{2}}{N}\right]^{1 / 2} .
\end{aligned}
$$

In (1), $\overline{\mathrm{PR}}$ and $\overline{\mathrm{KOUN}}$ represent the arithmetic mean of the PR and KOUN observations, respectively.

\section{Results and discussion}

\section{a. Comparisons of melting-layer height}

Scattering properties detected by polarimetric radar enable the classification of echoes based on their inferred sizes, shapes, phases, and orientations. TRMM PR is capable of retrieving melting-layer heights that can then be used to segregate precipitation into frozen, liquid, and mixed categories. Accurate depiction of the meltinglayer height or bright band is very important for accurate quantitative precipitation estimation, because reflectivity is known to be sensitive to large, wetted hydrometeors within this zone. Shin et al. (2000) described how the altitude of the melting layer from TRMM PR is estimated using the height of the maximum radar reflectivity and the largest positive and negative vertical gradients of reflectivity. Harris et al. (2000) compared a global "climatology" of TRMM melting-layer altitude estimates with the height of the $0^{\circ} \mathrm{C}$ isotherm from the National Centers for Environmental Prediction (NCEP) reanalysis dataset. They found that differences between TRMM and NCEP heights typically ranged from approximately -300 to $-900 \mathrm{~m}$. In this study, we extract PR brightband-height data directly from TRMM product 2A23. The melting-layer heights are recorded as a function of latitude and longitude and then are remapped to a 2D Cartesian grid having 2-km horizontal resolution. This chosen gridcell resolution oversamples the TRMM $2 \mathrm{~A} 23$ product that has $4-5-\mathrm{km}$ horizontal resolution but generally undersamples KOUN observations.

For KOUN, the approximate melting-layer height for each event is computed by averaging the heights of all bins classified by the HCA as "wet snow" (typical of radar bright bands) for elevation angles of $4^{\circ}-10^{\circ}$ (Giangrande et al. 2008; Park et al. 2009). The use of radar data exclusively from these higher elevation angles provides a more accurate estimate of the melting-layer height than would be possible from data collected at lower elevation angles. These bins are stored in spherical coordinates centered on KOUN as a function of range, azimuth, and elevation angle. The heights of the wet snow bins are then calculated assuming a $4 / 3$ Earth radius model to account for standard beam refraction. Then, the spherical coordinates are remapped to the same $2 \mathrm{D}$ Cartesian grid that contains the TRMM PR melting-layer heights, thus enabling their intercomparison. As such, we do not need to employ the 3D volume-matching technique described in section $2 \mathrm{~b}$ for melting-layer heights. It is noted that event 10 is a mesoscale convective system (MCS) case with intensive convection but lacking an extensive stratiform region, and therefore no bright band is detected from this event (as noted in Table 1). Comparisons of meltinglayer height are thus made for the remaining 19 events. Giangrande et al. (2008) developed a melting-layerdesignation algorithm using KOUN data on some of the same cases studied herein. They found that the algorithm successfully retrieved the $0^{\circ} \mathrm{C}$ height in $85 \%$ of the cases with a root-mean-square error of $0.22 \mathrm{~km}$. They also cited azimuthal and spatial variability of the KOUN-depicted melting layer as the cause of the discrepancies in comparing with $0^{\circ} \mathrm{C}$ isotherm heights from radiosonde observations and model analyses. Next, we compare TRMM PR with KOUN melting-layer heights, both of which should capture the spatial variability of the melting layer for matched data points.

Figure 2 shows results from the comparison of meltinglayer heights. The colored data-density scatterplot in Fig. 2a and the histograms in Fig. 2b indicate that there is good overall agreement, with a relative bias of $5.94 \%$ and a correlation coefficient of 0.88 . Both plots show that there is more scatter with the KOUN-detected meltinglayer heights than from TRMM PR. The wider range of KOUN melting-layer heights is caused by the relatively coarse vertical resolution from horizontally scanning platforms in comparison with the vertical scanning of TRMM PR, which has a height resolution of $0.25 \mathrm{~km}$ at nadir. For KOUN, the vertical resolution depends on range and elevation angle of beams that intercept the melting layer. We find that TRMM PR typically has a single value for melting-layer height within a nominal 2-km grid cell, whereas KOUN provides a range of heights as a result of radar beams increasing in altitude with range within the grid cell.

To assess a potential bias between the TRMM PR and KOUN melting-layer heights, we compute event-averaged melting-layer heights to minimize the impacts from different scanning orientations. A majority of events shown in Fig. 3a lie very close to the 1:1 line, although there are three events for which TRMM melting-layer heights are $1-1.5 \mathrm{~km}$ lower than that detected by KOUN. These points were associated with events 12,18 , and 19 , which are all strong convective warm-season events during June (see Table 1). In these events, the melting layer is 

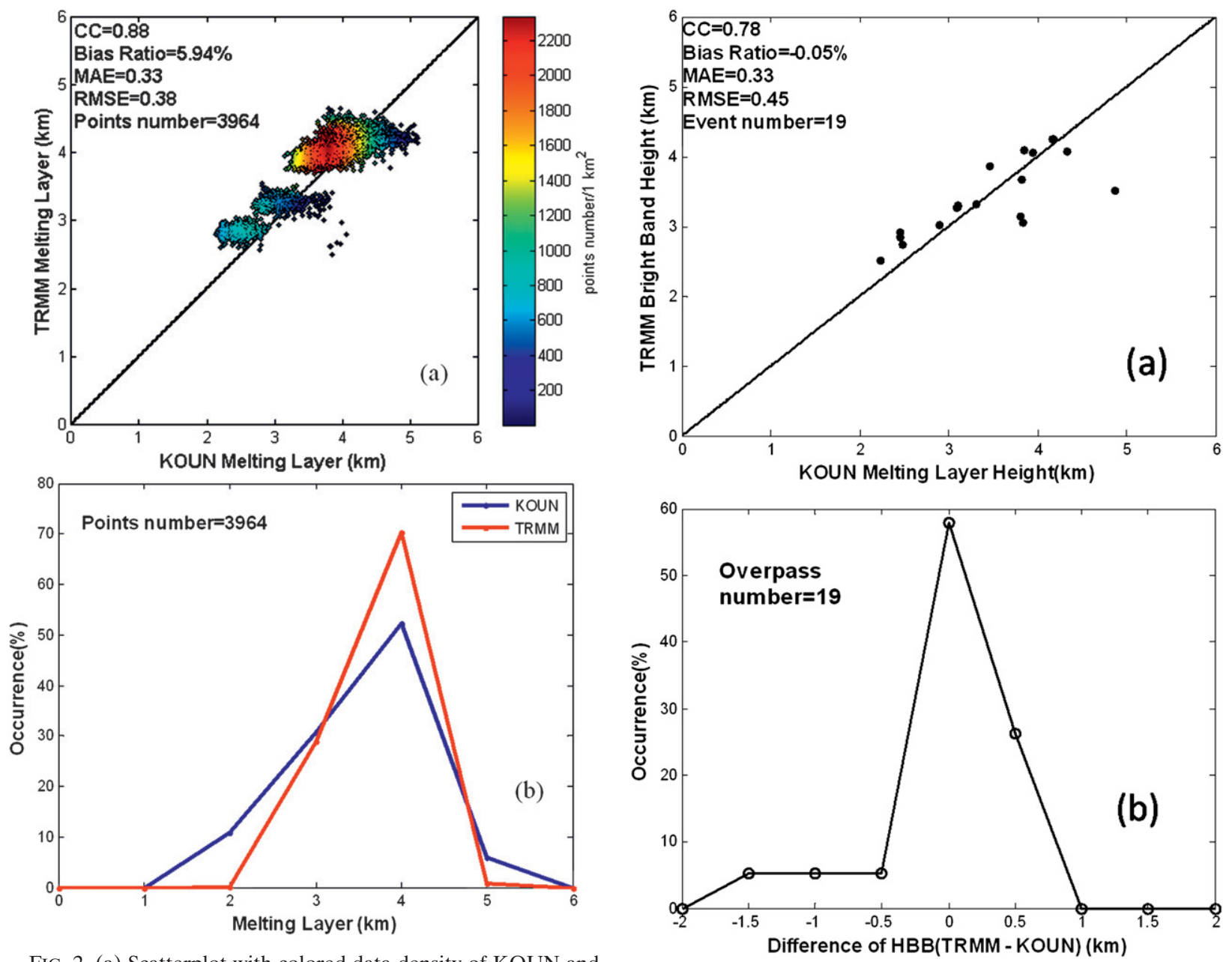

FIG. 2. (a) Scatterplot with colored data density of KOUN and TRMM 2A23 melting-layer heights for every pixel in 19 events. The correlation coefficient, bias ratio, MAE, RMSE, and sample size are shown in the embedded text. (b) Histograms of KOUN and TRMM 2A23 melting-layer heights for data shown in (a).

hardly discernible with $<12$ data pairs. The histogram of the difference in TRMM PR and KOUN melting-layer heights in Fig. $3 \mathrm{~b}$ also shows that a majority of points are close to $0-\mathrm{km}$ difference, indicating very little to no bias in detected melting-layer heights between the two instruments. In summary, the melting-layer height comparison reveals differences that are primarily explained from sampling differences between the two instruments rather than from systematic offsets that might have pointed to algorithmic errors.

\section{b. Reflectivity comparisons}

Using KOUN as a reference, comparisons of reflectivity $Z$ between the PR and KOUN can be used to assess the PR's performance. For each event shown in Table 1 , KOUN $Z$ data are compared with the neighboring KTLX NEXRAD radar in Twin Lakes, Oklahoma, which

FIG. 3. (a) Scatterplot of event-averaged melting-layer heights from KOUN and TRMM 2A23. (b) Histogram of melting-layer height difference of data shown in (a).

is known to have a stable calibration to within $1 \mathrm{~dB}$ as shown in Ryzhkov et al. (2005) and Giangrande and Ryzhkov (2005). KOUN differential reflectivity $Z_{\mathrm{DR}}$ is also manually calibrated for each event to within $0.2 \mathrm{~dB}$ by examining dry aggregated snow above the melting layer at elevation angles between $4.5^{\circ}$ and $6^{\circ}$. Here, the true or intrinsic $Z_{\mathrm{DR}}$ is known to be approximately $0.3 \mathrm{~dB}$ (Cao et al. 2008). Out of the 20 events shown in Table 1 , we compare 18 events that all have sufficient overlapping areas of coincident data coverage.

Figure 4 shows reflectivity comparisons between KOUN and TRMM 2A25 subject to subsequently applied qualitycontrol procedures. Figures $4 \mathrm{a}$ and $4 \mathrm{~b}$ show a colored datadensity scatterplot and histograms for reflectivity data with no quality control. A major advantage of polarimetric radar is its capability of distinguishing nonmeteorological echoes from meteorological echoes. Nonmeteorological echoes identified by the HCA, which contaminate radar 

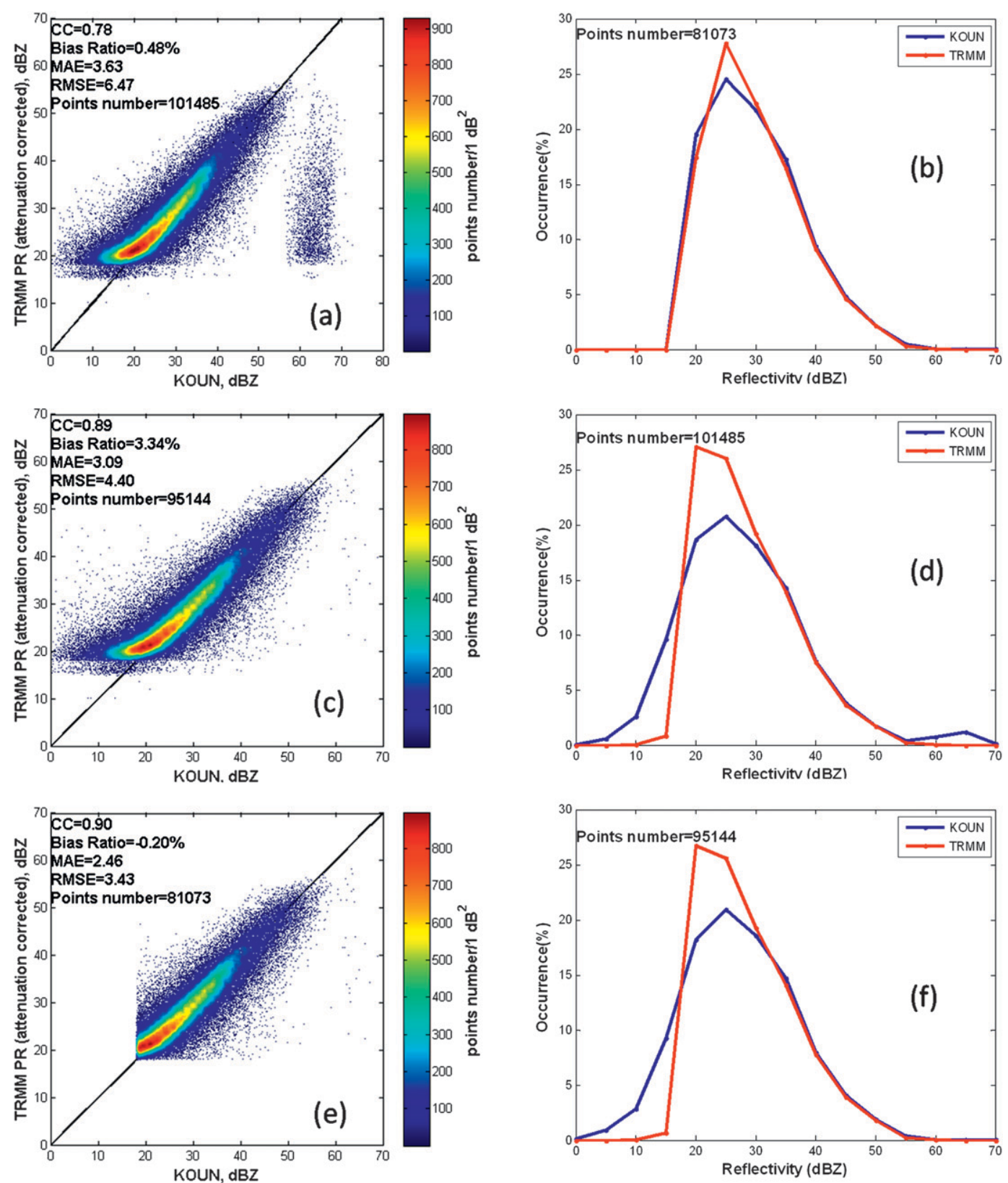

FIG. 4. Scatterplots with colored data density and histograms of KOUN and TRMM PR reflectivity. (a),(b) reflectivity and attenuation-corrected PR reflectivity with no additional quality control; (c),(d) as in (a) and (b), but nonmeteorological echoes based on KOUN's HCA have been removed; (e),(f) as in (c) and (d), but applied with a minimum $18-\mathrm{dB} Z$ threshold.

observations, have been removed from the KOUN and PR comparisons in Figs. 4c and 4d. Also, because of PR's low sensitivity of $18 \mathrm{~dB}$ or lower (NASDA 1999), only $Z>18 \mathrm{~dB} Z$ are used in comparing values in Figs. $4 \mathrm{e}$ and $4 \mathrm{f}$.

We can see substantial improvements in the reflectivity comparisons following the removal of nonmeteorological echoes and application of the $18-\mathrm{dB} Z$ threshold in the plots and statistics. The CC improves from 0.78 to 0.89 following removal of nonmeteorological echoes, and the RMSE decreases from 6.47 to 4.40 dB. In Fig. 4a, there is a separate cluster of points for which KOUN shows values of $Z$ from 55 to 70 corresponding to a much larger range of PR $Z$ values. These points are associated with nonmeteorological KOUN echoes, most likely resulting from ground clutter. In Fig. 4c, this cluster has been effectively removed following application of the HCA, with 


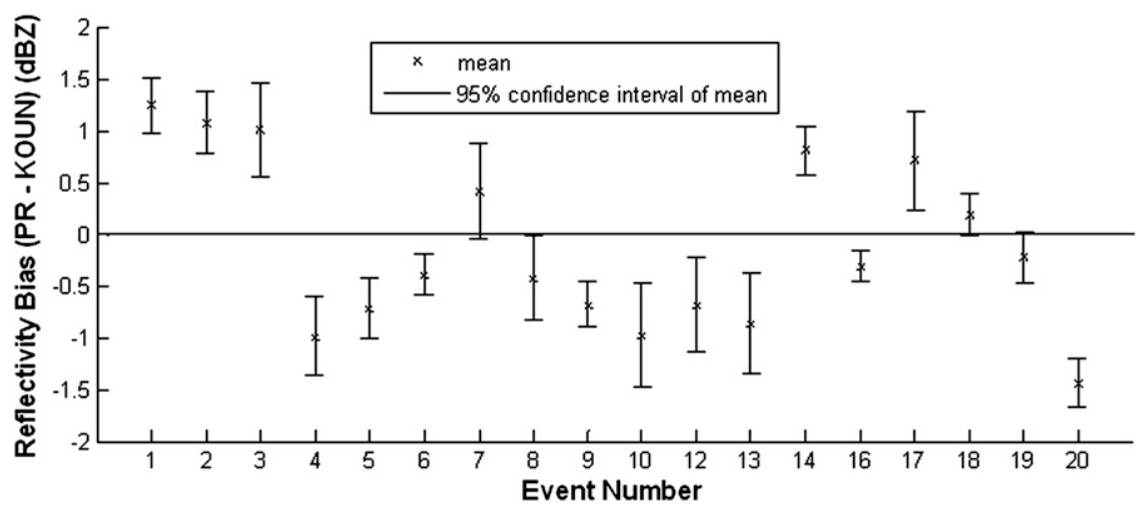

FIG. 5. Reflectivity bias between PR and KOUN for each event. Event details are provided in Table 1.

only a few points left. The histogram in Fig. 4d no longer contains the discrepancy in the occurrence of $Z$ in the range of $55-70 \mathrm{~dB} Z$.

The best results are shown in Figs. $4 \mathrm{e}$ and $4 \mathrm{f}$ with both nonmeteorological echoes removed and the $18-\mathrm{dBZ}$ threshold applied. Following application of the threshold, the sample size is reduced from 95144 to 81073 points, or $-14.8 \%$. Thus, it can be inferred that KOUN is capable of detecting rain for this fraction of light rain when PR is not. Using the same quality-controlled data shown in Figs. 4 e and 4f, Fig. 5 shows the mean reflectivity bias for each event with $95 \%$ confidence intervals provided as vertical error bars. We construct a Student's $t$-test statistic (Wilks 1995) to derive the $100(1-\alpha) \%$ confidence interval of the mean reflectivity bias:

$$
\left(\overline{\operatorname{bias}}-t_{\alpha / 2} \frac{s}{\sqrt{n}}, \overline{\operatorname{bias}}+t_{\alpha / 2} \frac{s}{\sqrt{n}}\right),
$$

where $\alpha$ is a statistical significance level of $5 \%, t_{\alpha / 2}$ is the $97.5 \%$ percentile of the $t$ distribution with $n-1$ degrees of freedom, $n$ is the sample size, and $s$ is the sample standard deviation of the individual reflectivity bias values as follows:

$$
s=\left[\frac{1}{n-1} \sum_{n}(\text { bias }-\overline{\text { bias }})^{2}\right]^{1 / 2} .
$$

Figure 5 shows that the event-to-event reflectivity bias between PR and KOUN varies by less than $1.5 \mathrm{~dB}$. Although remaining differences shown in Figs. 4e, 4f, and 5 are slight, about as small as can be expected between two independent remote sensing instruments, it is possible that remaining discrepancies could be related to difficult-to-discern random factors, such as spatial and temporal volume mismatches or nonuniform beam-filling effects. It is also possible that the discrepancies may be due to systematic, nonrandom effects such as errors in the PR attenuation correction scheme and differences in backscattered radiation between PR and KOUN at 2.17- and 10.7- $\mathrm{cm}$ wavelength, respectively (Bolen and Chandrasekar 2000; Liao and Meneghini 2009; Schumacher and Houze 2000; Wang and Wolff 2009). These potential nonrandom factors are elucidated in the following section with the aid of HCA results.

\section{c. Reflectivity comparisons for hydrometeor types}

Non-Rayleigh scattering effects are significant for TRMM PR at a frequency of $13.8 \mathrm{GHz}$. For KOUN S-band radar, however, nearly all hydrometeors in our dataset satisfy the Rayleigh approximation. Because of different backscattering cross sections measured by the radars, $P R Z$ can be up to $2 \mathrm{~dB}$ higher than that of KOUN for rain measurements in the range of $40-50 \mathrm{dBZ}$ (Bolen and Chandrasekar 2000). Other types of hydrometeors (e.g., dry aggregated snow, wet snow, or mixture of rain and hail) have different backscattering cross sections at $\mathrm{Ku}$ and $\mathrm{S}$ band, however, potentially resulting in systematic discrepancies between PR and KOUN observations.

The difference in $Z$ at $\mathrm{S}$ and $\mathrm{Ku}$ bands for different hydrometeors is simulated and is shown in Fig. 6. The radar reflectivity factor $Z$ is given as

$$
Z=\frac{\lambda^{4}}{\pi^{5}\left|K_{W}\right|^{2}} \int_{0}^{\infty} N(D) \sigma_{b} d D,
$$

where $K_{w}=\left(m^{2}-1\right) /\left(m^{2}+2\right)$ and $m$ is the complex refractive index of water (Doviak and Zrnic 1993). We choose 0.93 for $\left|K_{w}\right|^{2}$. Here, $N(D)$ is the particle size distribution, which is simulated by a monodispersion model using a number concentration of $1 \mathrm{~m}^{-3}$. The $\sigma_{b}$ is the backscattering cross section of hydrometeors that is simulated using the T-matrix method (Waterman 1971; 


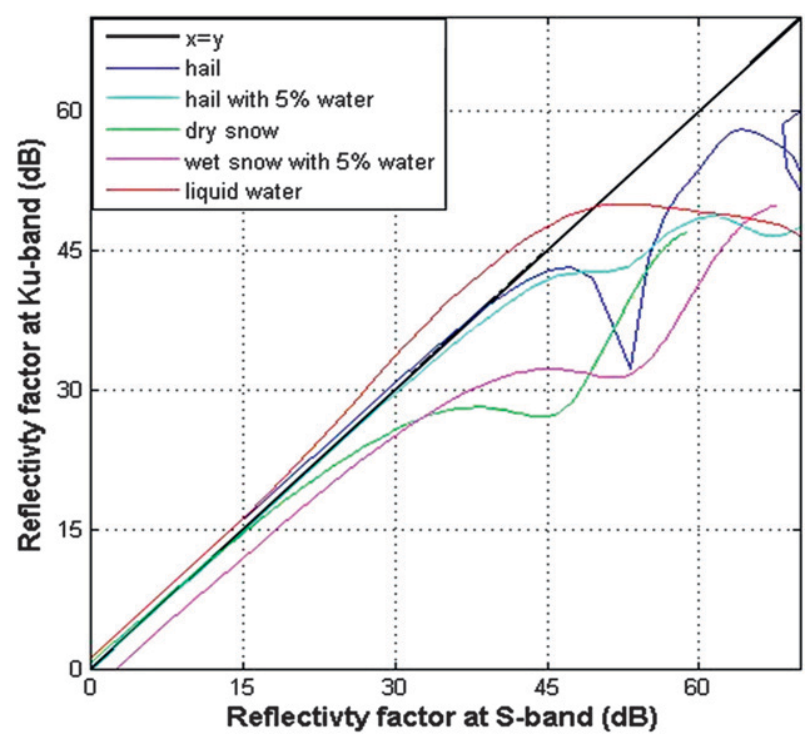

FIG. 6. Relationships between reflectivity factors at $\mathrm{S}$ and $\mathrm{Ku}$ bands for liquid water, hail, and dry snow. Backscatter cross sections are simulated using the T-matrix method at $\mathrm{S}$ and $\mathrm{Ku}$ bands, where reflectivity calculations assume a monodispersed drop size distribution.

Vivekanandan et al. 1991) at different radar wavelengths $\lambda$. As seen in Fig. 6, $Z$ values at horizontal polarization at $\mathrm{Ku}$ and $\mathrm{S}$ band are approximately equal for all hydrometeor types for $Z<30 \mathrm{~dB} Z$, with the exception of wet snow, which has lower $Z$ at Ku band. As $Z$ increases above $30 \mathrm{~dB} Z$, all hydrometeors except liquid water have lower reflectivity at Ku band because of nonRayleigh scattering effect. For liquid water, the simulated reflectivity gradually deviates for $Z$ in the range of 40-50 dBZ, which conforms to results shown in Bolen and Chandrasekar (2000). Beyond $50 \mathrm{dBZ}$, the deviation becomes more severe. Liao and Meneghini (2009) also show that PR underestimates in heavy rain.

The HCA discerns eight different classes of hydrometeors based on polarimetric characteristics of the radar echoes. The analysis in section 3a indicates PR and KOUN retrieve comparable melting-layer heights; thus we can confidently classify different hydrometeor classes exclusively based on the KOUN HCA. During the PR-KOUN volume-matching procedure, we calculate the percentage of each hydrometeor class in each volume. The final hydrometeor class is assigned to a bin if a particular hydrometeor type has the highest percentage in a volume and the percentage exceeds $50 \%$. We then group all KOUN-PR reflectivity data with respect to the different hydrometeor types.

The reflectivity comparisons classified by different hydrometeor types are shown in Fig. 7. Very few classifications are made in the HCA category of "crystals of various orientations," or pristine ice, which may be specific to our dataset or perhaps indicates a failure in HCA itself. The scatterplots with colored data density and histograms for reflectivity in rain-hail mixture, wet snow, graupel, and heavy rain show that they have an obvious negative bias at $\mathrm{Ku}$ band, which is in good qualitative agreement with simulated results shown in Fig. 6. The reflectivity comparison of light and moderate rain types (Figs. 7f1,7f2) also conforms to expectations shown in Fig. 6 with very little bias between $\mathrm{Ku}-$ and S-band reflectivity. For dry snow with snow mass densities of $0.1 \mathrm{~g} \mathrm{~cm}^{-3}$, however, simulations indicate that PR $Z$ should be less than KOUN, especially for values of $Z>25 \mathrm{dBZ}$. Figures $7 \mathrm{~g} 1$ and $7 \mathrm{~g} 2$ do not reveal this discrepancy in the observations, with a relative bias of only $1.92 \%$. Liao and Meneghini (2009) also show that simulated reflectivity at $\mathrm{S}$ band should be higher than at $\mathrm{Ku}$ band for snow by assuming the Gunn-Marshall (Gunn and Marshall 1958) snow particle size distribution with a snow density of $0.3 \mathrm{~g} \mathrm{~cm}^{-3}$. Their (and our) quantitative bias for snow $Z$ between $\mathrm{S}$ band and $\mathrm{Ku}$ band does not clearly agree with the expected bias shown in the simulation, however. It is possible that assumptions used in the simulations such as the dry snow density and particle size distribution differ from observations-an area that invites future research. Another possible explanation for the discrepancy in horizontally polarized $\mathrm{Z}$ observations and modeling results with dry snow is the impact of different viewing geometries (approximately vertical vs horizontal). For instance, it is possible that asymmetrical frozen hydrometeors identified as dry snow, such as needles, become aligned in the presence of a wind or electric field. This alignment would result in a bias in reflectivity at horizontal polarization between horizontal and vertical scanning perspectives. Further insights can be gained by examining the azimuthal dependence of $Z_{\mathrm{DR}}$ observations from KOUN in these regions.

A simple bar chart in Fig. 8 gives an overview of the PR reflectivity bias as a function of the different hydrometeor types. Rain-hail mixture, wet snow, graupel, heavy rain, and big drops show the largest negative biases, moderate rain observations are unbiased, and dry snow indicates a positive PR bias. Differences in scattering between $\mathrm{Ku}$ and $\mathrm{S}$ band are the main reason to explain the discrepancies, as confirmed in the T-matrix simulations. Simulation of multifrequency reflectivity values for dry snow is an area that invites future research.

\section{d. Vertical profile of reflectivity comparisons}

A method to identify potential attenuation correction errors applied to TRMM PR reflectivity data is to compare vertical profiles of reflectivity from PR with 

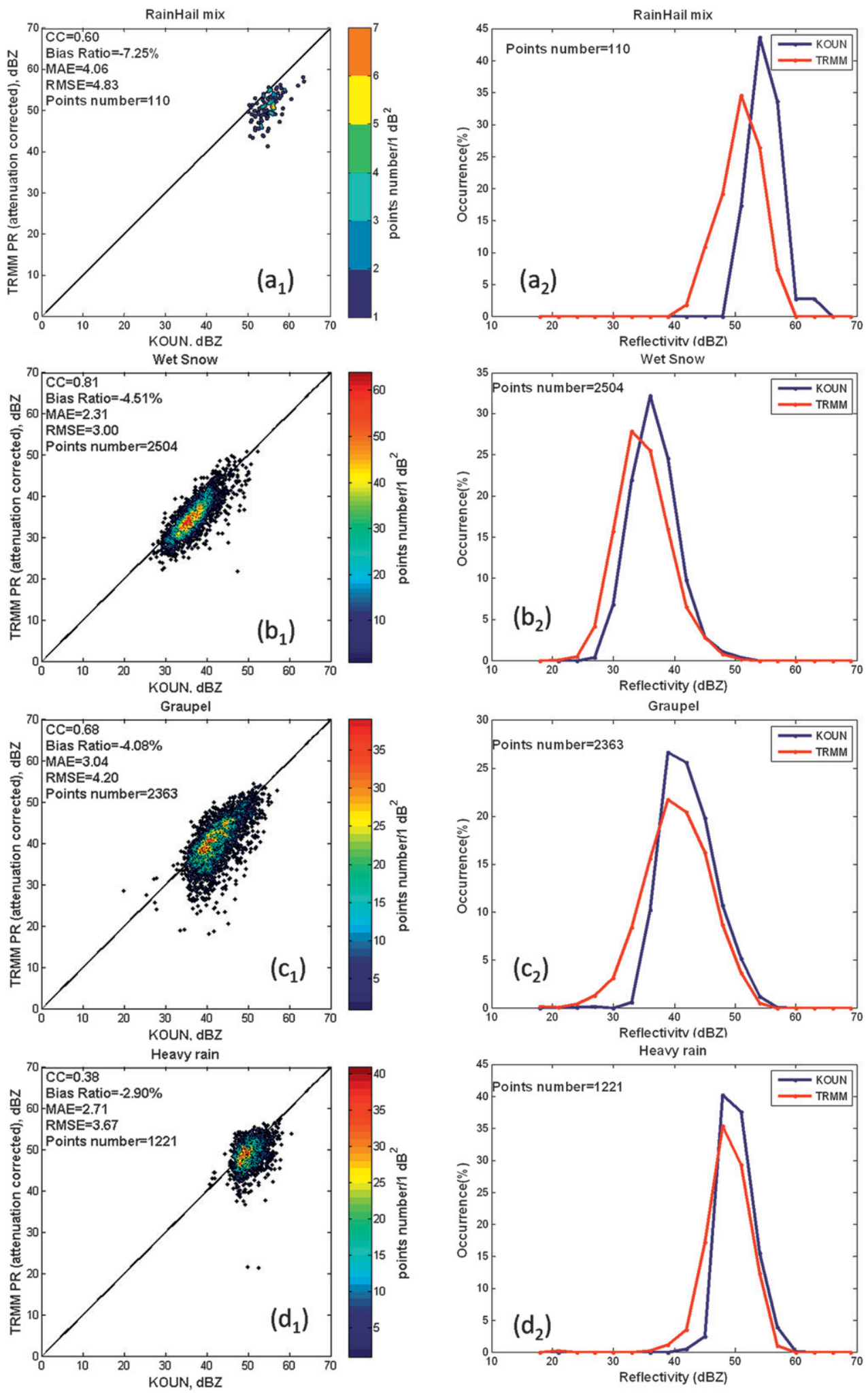

FIG. 7. Scatterplots with colored data density in the first column and histograms in the second column for reflectivity observed for different hydrometeors as determined from KOUN HCA: (a) rain-hail mix, (b) wet snow, (c) graupel, (d) heavy rain, (e) big drops, (f) moderate rain, and (g) dry snow. 

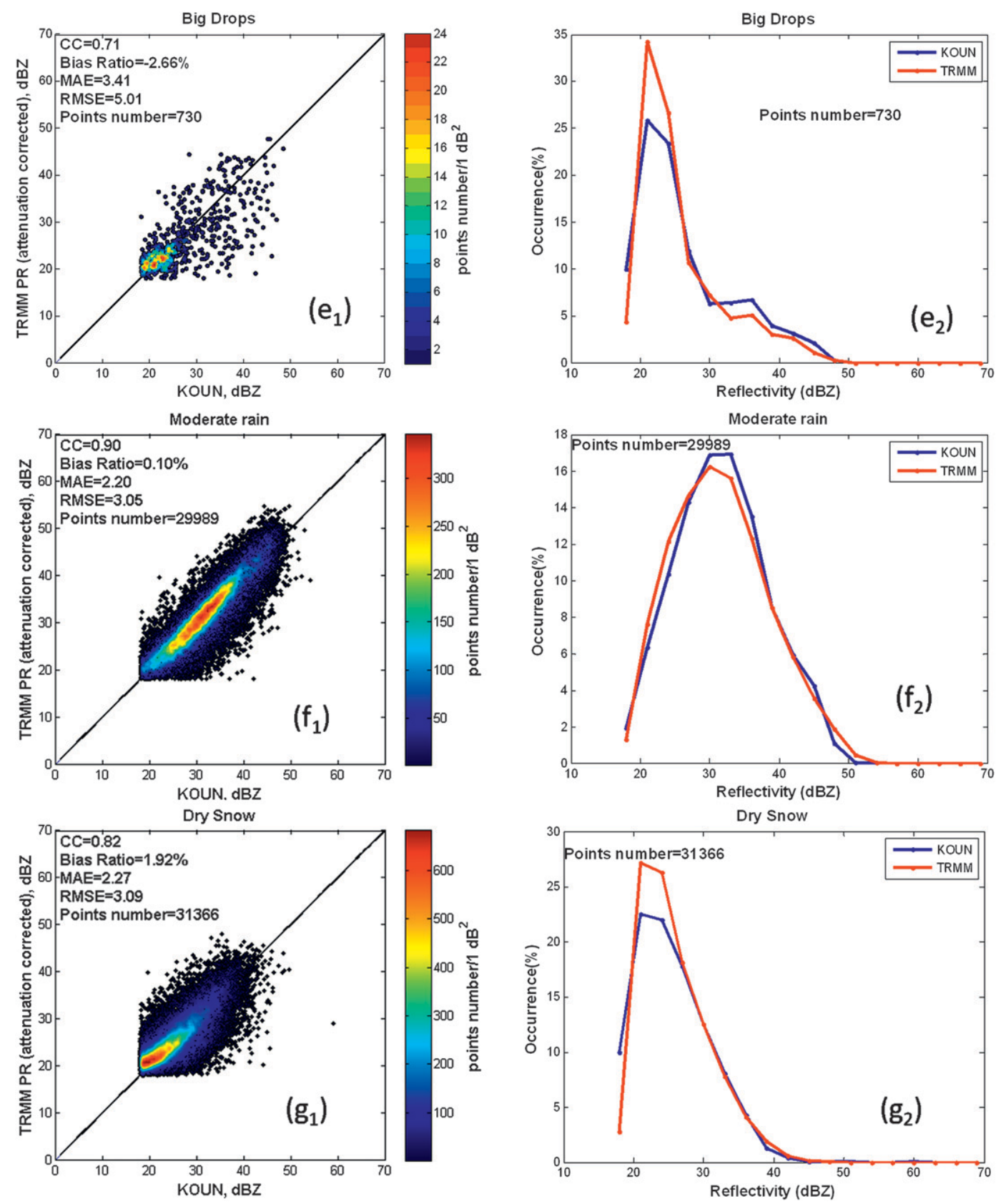

FIG. 7. (Continued)

those of KOUN, where KOUN is again the reference. A hybrid correction scheme combining the surface reference technique and the Hitschfeld and Bordan method is used in the PR attenuation correction (Iguchi et al. 2000). The magnitude of the correction increases with pathlength; thus it is informative to compare reflectivity profiles in the vertical, path-integrated direction. Figure 9 shows the average profiles for convective, stratiform, and all rain types combined using all of the quality-controlled, matched data shown in Figs. 4e and 4f, totaling 81073 points. The rain-type classification is made exclusively according to PR observations, which are from the TRMM $2 \mathrm{~A} 23$ product. To reveal the magnitude of estimated attenuation losses, the PR-measured reflectivity ( $\mathrm{Zm}$; uncorrected reflectivity from $1 \mathrm{C} 21)$ is shown in the profiles along with corrected PR reflectivity ( $\mathrm{Zc}$; attenuation-corrected reflectivity from 2A25) and the reference reflectivity from the KOUN ground radar $(\mathrm{ZG})$. The profiles are computed at nine vertical layers from 1.5 to $13.5 \mathrm{~km}$ with $1.5-\mathrm{km}$ spacing 


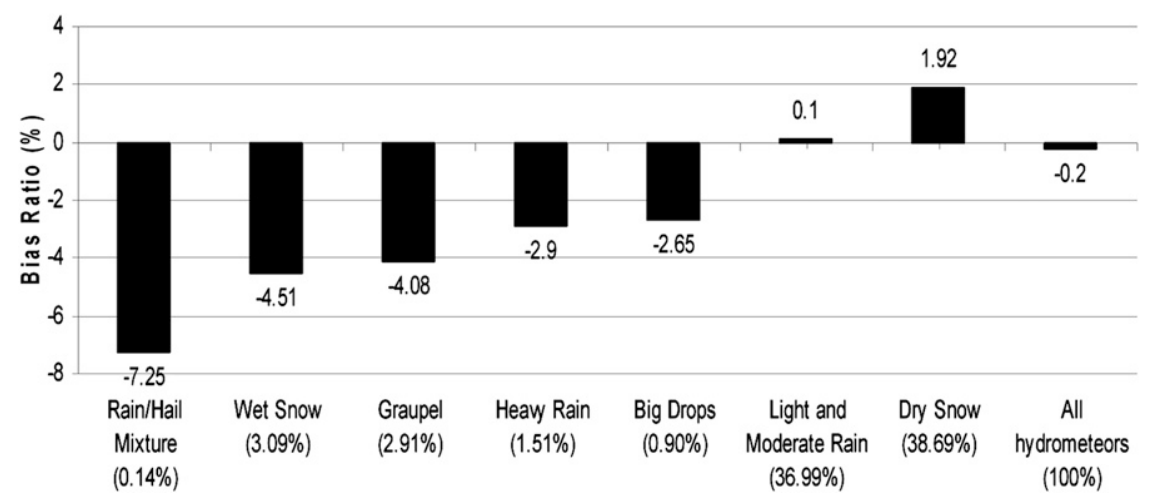

FIG. 8. Bias (\%) of TRMM PR reflectivity observations relative to KOUN for different hydrometeor types as discriminated by the dual-polarization HCA.

for values of $Z>18 \mathrm{~dB} Z$. As the pathlength increases from the top down in Fig. 9a, the gap between the $\mathrm{Zm}$ and $\mathrm{Zc}$ curves gradually increases, which illustrates that PR suffers significant attenuation losses. In comparing $\mathrm{Zc}$ with $\mathrm{ZG}$ in stratiform rain from 3 to $4.5 \mathrm{~km}$, we see that there is a large discrepancy in the presumed melting layer (Fig. 9b). The hydrometeors within the melting layer are primarily wet snow, and from results shown in Fig. 8 we can see that TRMM PR underestimates S-band reflectivity due to the effect of nonRayleigh scattering. At the lowest height of $1.5 \mathrm{~km}$, however, Zc and ZG converge, indicating that the attenuation correction for stratiform rain performs well at the surface. The same convergence of $\mathrm{Zc}$ and $\mathrm{ZG}$ values is also noted in convective precipitation at the surface level. As shown in Fig. 9c for all rain types combined, because of mismatches in resolution volumes and/ or different backscatter cross sections between PR and KOUN, the reflectivity profiles have slight discrepancies, but at the surface level the measurements agree very well. To summarize, our analysis shows close correspondence between corrected TRMM PR near-surface reflectivity observations and KOUN data, thus indicating that no systematic biases were caused by the TRMM attenuationcorrection procedures.

\section{Summary and conclusions}

This study provides a quantitative assessment of TRMM PR melting-layer and reflectivity measurements as compared with an S-band polarimetric radar located in Norman, Oklahoma. The KOUN ground-based radar is the prototype, proof-of-concept radar for the ongoing upgrade of the NEXRAD network. KOUN is shown to be particularly useful in this analysis because of its ability to filter nonmeteorological echoes and to discriminate hydrometeor species, each of which has differing scattering characteristics at $\mathrm{Ku}$ - and S-band frequencies. The main findings are summarized as follows:

1) Comparisons of TRMM PR and KOUN meltinglayer heights reveal a correlation coefficient of 0.88 and a relative bias of $5.94 \%$. The differences are deemed to be due to the vertical versus horizontal scanning and resolution-volume differences rather than systematic offsets that might have pointed to algorithmic errors.

2) NASA's resolution volume-matching technique is used to match and compare simultaneous TRMM PR and KOUN reflectivity observations. The results indicate a negligible bias $(<0.2 \%)$ due to calibration differences between PR and KOUN. For $Z>50 \mathrm{~dB} Z$, however, reflectivity from KOUN is slightly higher than that from PR, likely because of non-Rayleigh scattering for $\mathrm{Ku}$ band of PR.

3) By comparing reflectivity with respect to different hydrometeor types (as determined by KOUN's hydrometeor classification algorithm), we find biases are primarily from rain-hail mixture, wet snow, graupel, and heavy rain. These results agree with differences in simulated reflectivity differences at $\mathrm{Ku}$ and $\mathrm{S}$ band using the T-matrix method, with the notable exception of dry snow. Assumptions for simulating reflectivity in dry snow need to be further investigated.

4) Comparison of vertical reflectivity profiles shows that PR suffers significant attenuation, especially in convective rain and within the melting layer. TRMM PR observations correspond very closely with KOUN reflectivity measured nearest to the surface, however, thus indicating that no systematic biases are caused by the TRMM attenuationcorrection procedures.

NASA has called for comprehensive sensor calibration and ground-validation research to be conducted to 

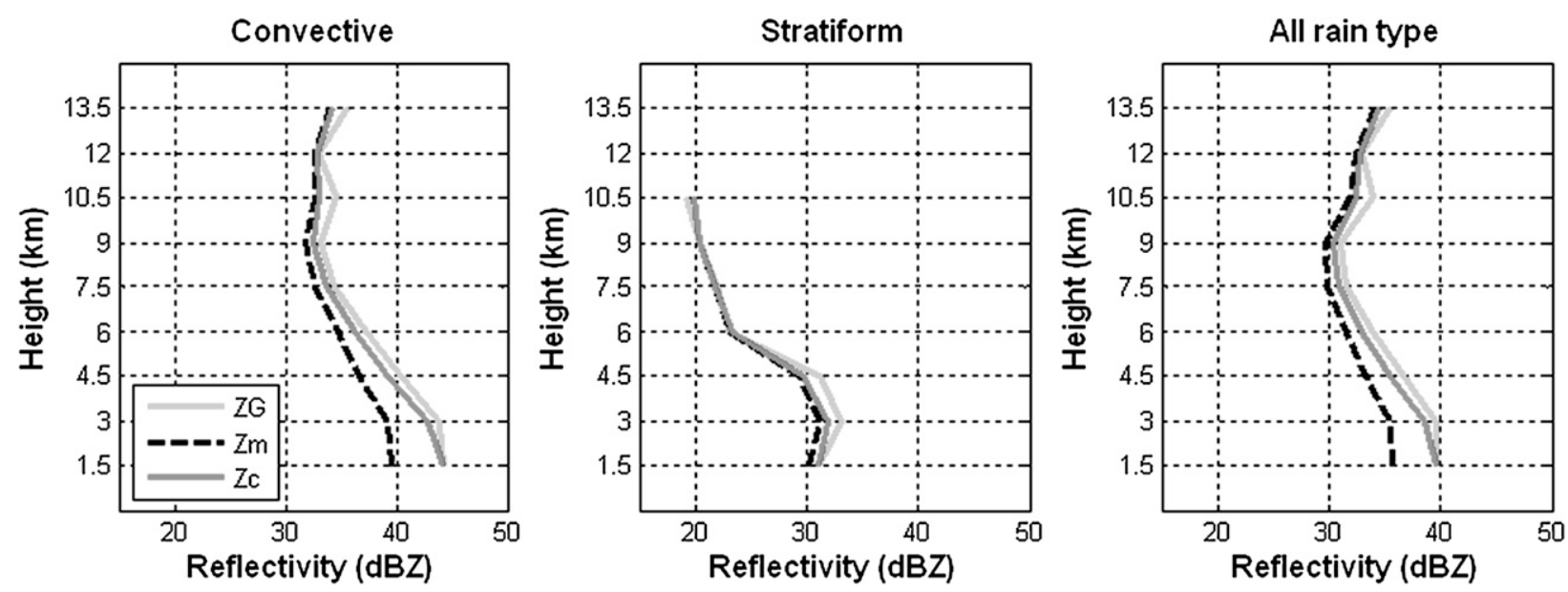

FIG. 9. Mean vertical reflectivity profiles constructed from 81073 quality-controlled points shown in Figs. $4 \mathrm{e}$ and $4 \mathrm{f}$ for (a) convective, (b) stratiform, and (c) all rain types as determined by TRMM 2A23. Here, ZG is reflectivity from KOUN, Zm is measured reflectivity from TRMM 1C21 with no attenuation correction, and $\mathrm{Zc}$ is attenuation-corrected TRMM 2A25.

ensure proper accuracy and precision of the spaceborne QPE missions (Petersen and Schwaller 2008). With the imminent upgrade of the U.S. national weather radar network to include polarimetric capabilities, the polarimetric algorithms developed on the prototype KOUN radar may be able to serve as the basis for a nationwide validation network using polarimetric NEXRAD data for NASA space QPE products. Benefits to the groundradar community from space-based radars include a stable and mobile radar source that can be used to calibrate NEXRAD instruments. Space-based observations can also be used to fill gaps in the NEXRAD coverage, which are significant in the intermountain west. Furthermore, this research motivates and invites synergistic development of multisensor rainfall algorithms using coordinated observations from space and ground.

Acknowledgments. This research was funded by Atmospheric Radar Research Center (ARRC) Seed Grant 2009 at the University of Oklahoma (http://arrc.ou.edu). We thank NASA Global Precipitation Measurement Ground Validation Science Manager Dr. Walter A. Petersen for his constructive comments and encouragement. We also are thankful for the support from NOAA/National Severe Storms Laboratory and University of Oklahoma Cooperative Institute for Mesoscale Meteorological Studies personnel who maintain and operate the KOUN polarimetric WSR-88D. The PR products used in this work were acquired as part of the activities of NASA's Science Mission Directorate and are archived and distributed by the Goddard Earth Sciences Data and Information Services Center (DISC). Constructive reviews provided by three anonymous reviewers improved the readability of this manuscript. Their time is appreciated.

\section{REFERENCES}

Amitai, E., X. Llort, and D. Sempere-Torres, 2009: Comparison of TRMM radar rainfall estimates with NOAA next-generation QPE. J. Meteor. Soc. Japan, 87A, 109-118.

Anagnostou, E., C. Morales, and T. Dinku, 2001: The use of TRMM precipitation radar observations in determining ground radar calibration biases. J. Atmos. Oceanic Technol., 18, 616-628.

Barnes, S. L., 1973: Mesoscale objective map analysis using weighted time series observations. NOAA Tech. Memo. ERL NSSL-62, $60 \mathrm{pp}$.

Bolen, S., and V. Chandrasekar, 2000: Quantitative cross validation of space-based and ground-based radar observations. J. Appl. Meteor., 39, 2071-2079.

— spaceborne radar and ground-based radar observations. J. Atmos. Oceanic Technol., 20, 647-659.

Cao, Q., G. Zhang, E. Brandes, T. J. Schuur, A. V. Ryzhkov, and K. Ikeda, 2008: Analysis of video disdrometer and polarimetric radar data to characterize rain microphysics in Oklahoma. J. Appl. Meteor. Climatol., 47, 2238-2255.

Chandrasekar, V., A. Hou, E. Smith, V. N. Bringi, S. A. Rutledge, E. Gorgucci, W. A. Petersen, and G. S. Jackson, 2008: Potential role of dual-polarization radar in the validation of satellite precipitation measurements: Rationale and opportunities. Bull. Amer. Meteor. Soc., 89, 1127-1145.

Doviak, R. J., and D. S. Zrnic, 1993: Doppler Radar and Weather Observations. 2nd ed. Dover, 562 pp.

Giangrande, S. E., and A. V. Ryzhkov, 2005: Calibration of dualpolarization radar in the presence of partial beam blockage. J. Atmos. Oceanic Technol., 22, 1156-1166.

, and - 2008: Estimation of rainfall based on the results of polarimetric echo classification. J. Appl. Meteor. Climatol., 47, $2445-2462$ 
— J. M. Krause, and A. V. Ryzhkov, 2008: Automatic designation of the melting layer with a polarimetric prototype of the WSR-88D radar. J. Appl. Meteor. Climatol., 47, 1354-1364.

Gunn, K. L. S., and J. S. Marshall, 1958: The distribution with size of aggregate snowflakes. J. Meteor., 15, 452-461.

Harris, G. N., Jr., K. P. Bowman, and D. B. Shin, 2000: Comparison of freezing-level altitudes from the NCEP reanalysis with TRMM precipitation radar brightband data. J. Climate, 13, 4137-4148.

Iguchi, T., R. Meneghini, J. Awaka, T. Kozu, and K. Okamoto, 2000: Rain profiling algorithm for TRMM precipitation radar. J. Appl. Meteor., 39, 2038-2052.

Kozu, T., and Coauthors, 2001: Development of precipitation radar onboard the Tropical Rainfall Measuring Mission (TRMM) satellite. IEEE Trans. Geosci. Remote Sens., 39, 102-116.

Liao, L., and R. Meneghini, 2009: Validation of TRMM precipitation radar through comparison of its multiyear measurements with ground-based radar. J. Appl. Meteor. Climatol., 48, 804-817.

Morris, K. R., and M. R. Schwaller, 2009: An enhanced global precipitation measurement (GPM) validation network prototype. Preprints, 34th Conf. on Radar Meteorology, Williamsburg, VA, Amer. Meteor. Soc., P7.3. [Available online at http://ams. confex.com/ams/pdfpapers/155254.pdf.]

NASDA, 1999: TRMM PR algorithm instruction manual V1.0. Communications Research Laboratory, 52 pp. [Available from Communications Research Laboratory, 4-2-1 Nukuikitamachi, Koganei-chi, Tokyo 184, Japan.]

Park, H., A. V. Ryzhkov, D. S. Zrnic, and K. E. Kim, 2009: The hydrometeor classification algorithm for the polarimetric
WSR-88D: Description and application to an MCS. Wea. Forecasting, 24, 730-748.

Petersen, W. A., and M. R. Schwaller, 2008: Global Precipitation Mission (GPM) ground validation science implementation plan (draft). NASA Doc., 37 pp. [Available online at http://gpm. gsfc.nasa.gov/documents/GPM_GVS_imp_plan_Jul08.pdf.]

Ryzhkov, A. V., S. E. Giangrande, V. M. Melnikov, and T. J. Schuur, 2005: Calibration issues of dual-polarization radar measurements. J. Atmos. Oceanic Technol., 22, 1138-1155.

Schumacher, C., and R. A. Houze, 2000: Comparison of radar data from the TRMM satellite and Kwajalein oceanic validation site. J. Appl. Meteor., 39, 2151-2164.

Shin, D.-B., G. R. North, and K. P. Bowman, 2000: A summary of reflectivity profiles from the first year of TRMM radar data. J. Climate, 13, 4072-4086.

Simpson, J., C. Kummerow, W.-K. Tao, and R. F. Adler, 1996: On the Tropical Rainfall Measuring Mission (TRMM). Meteor. Atmos. Phys., 60, 19-36.

Vivekanandan, J., W. M. Adams, and V. N. Bringi, 1991: Rigorous approach to polarimetric radar modeling of hydrometeor orientation distributions. J. Appl. Meteor., 30, 1053-1063.

Wang, J., and D. Wolff, 2009: Comparisons of reflectivities from the TRMM precipitation radar and ground-based radars. J. Atmos. Oceanic Technol., 26, 857-875.

Waterman, P. C., 1971: Symmetry, unitarity, and geometry in electromagnetic scattering. Phys. Rev. D, 3, 825-839.

Wilks, D. S., 1995: Statistical Methods in the Atmospheric Sciences: An Introduction. Academic Press, 467 pp. 